lected along the Little Buffalo River, about one mile below the mouth of the Sass River on May 26 (specimen deposited in Canadian Wildlife Service collection at Fort Smith). Two others were seen on June 18 along a cattail slough near the Little Buffalo River, about four miles downstream from the mouth of the Sass River. These records may represent the northernmost of any reptile in North America.

\section{AMPHIBIANS}

Boreal Chorus Frog Pseudacris triseriata maculata. Choruses of this species were heard throughout the Grand Detour Portage area and along the Little Buffalo River during the period May 23-30.

Leopard Frog Rana pipiens. Robert P. Allen reported seeing Leopard Frogs near the mouth of the Sass River on May 26.

Wood Frog Rana sylvatica. Common in moist situations along the Little Buffalo and Sass Rivers. Freshly laid eggs were seen along the Great Slave Lake Shore (near mouth of Little Buffalo River) on May 29.

\section{Notes on Butterflies}

*RED-Disked AlPine Erebia discoidalis. Fairly common in prairies and sedge meadows along the Sass River (about two miles above its mouth during period June 5-10).

* FreiJa Fritillary Boloria freija. Fairly common in prairies and sedgemeadows along the Sass River during period May 26-June 10.

*Saga Fritillary Boloria frigga. Fairly common in prairies and sedge- meadows along the Sass River during period June 5-10.

Pearl Crescent Phyciodes tharos. One seen on prairie between Slave and Little Buffalo Rivers on June 18.

*Hoary Comma Polygonia gracilis. Common along Sass River during period June 5 - 17 .

* Mourning Cloak Nymphalis antiopa. Fairly common along Little Buffalo and Sass Rivers during period May 27 - June 17.

BANDED PURPLE Limenitis arthemis. One seen at Fort Smith on June 21.

* Silvery Blue Glaucopsyche lygdamus. Common along Sass River during period June 5-17.

* SPring AzUre Lycaenopsis argiolus. Common along Little Buffalo and Sass Rivers during period May 26 June 17.

*Tiger Swallowtail Papilio glaucus. Fairly common along the Sass River during period June 5-17.

*AUsonides Euchloe ausonides. One was collected on a small prairie on June 12.

*Mustard White Pieris napi. Common throughout the area from June $1-17$.

*Dreamy Dusky Wing Erynnis icelus. Fairly common near the brushy margins of prairies and sedgemeadows, along the Sass River during the period June $5-17$.

Note-A few butterflies of the genus Colias and one of the genus Speyeria were also observed, but not identified to species.

* Specimen collected.

\title{
TWENTY-FOURTH ANNUAL SASKATCHEWAN CHRISTMAS BIRD COUNT, 1965
}

One could almost use last year's summary again this year, since the 1965 Christmas Bird Count almost duplicates that of 1964. A total of 28 localities reported, 26 from Saskatchewan and one from each of Alberta and the Northwest Territories. Extremely cold weather prevailed during most of the count period. Of the 26 Saskatchewan counts, 18 were taken in sub-zero weather and seven of those at $-20^{\circ}$ or lower. Bangor reported the lowest Saskatchewan temperature with $-34^{\circ}$. This was exceeded only by Cambridge Bay, N.W.T., where Ramon Burron and Rowan 
Raphael braved $-40^{\circ}$ (wind chill $-70^{\circ}$ ) for $3 \frac{1}{2}$ hours to compile their count.

Fifty-one species were recorded on "count" days with five others seen during the count period. No new species were added to the all-time list, so the total remains at 111 . There seemed to be little change in the number of locations in which any of the sighted species was found. Black-billed Magpie (25 of 26 Saskatchewan localities), House Sparrow (21/26), Black-capped Chickadee $(20 / 26)$ and Snow Bunting $(20 / 26)$ were the most frequently reported species. The Regina Natural History Society again reported the largest number of species with a total of 31 for the "count day" plus six additionals during "count period".

Manitoba counts contributed to the Blue Jay are forwarded to the Newsletter of the Manitoba Natural History Society for publication.

BANGOR, Sask. Jan. 2, 1966; 14 miles by car and around farm yard; temp. $-34^{\circ}$; wind light. 8 species, 217 individuals. Sharp-tailed Grouse, 1; Gray Partridge, 7; Downy Woodpecker, 2; Black-billed Magpie, 2; Black-capped Chickadee, 5; House Sparrow, 25; Evening Grosbeak, 15; Snow Bunting, 150.-Mrs. A. Thompson.

BATTLEFORD, Sask. Dec. $31 ; 5$ miles on foot in $3 \frac{1}{2}$ hours; temp. $-12^{\circ}$; wind NW $5 \mathrm{mph} ; 12$ inches of snow. 12 species, 420 individuals. Bald eagle, 1; Ruffed Grouse, 3; Sharp-tailed Grouse, 2; Rock Dove, 2; Great Horned Owl, 1; Black-billed Magpie, 2; Black-capped Chickadee, 1 ; Bohemian Waxwing, 163; House Sparrow, 8; Evening Grosbeak, 3; Pine Grosbeak, 34; Common Redpoll, 200. (Add: Golden Eagle, 1, Dec. 23; Hairy Woodpecker, 2, Dec. 24; Downy Woodpecker, 1, Dec. 24.)Harold Fisher, Glen A. Fox, Spencer G. Sealy (compiler).

BLADWORTH, Sask. Dec. 25; 25 miles by car. 4 species, 1053 individuals. Sharp-tailed Grouse, 26; Gray Partridge, 7; Rock Dove, 20; Snow Bunting, 1000. (Add: Golden Eagle,
1, Dec. 21; Snowy Owl, 1, Dec. 21; Horned Lark, 2 on Dec. 21, 6 on Jan. 1; Black-billed Magpie, 15, Dec. 23; Common Redpoll, 70, Dec. 31.)-Mr. and Mrs. Lawrence Beckie, Theresa, Bill, Gary and Hugh Beckie.

CARON, Sask. Dec. 26; at feeding station and 7 miles by car; temp. $-16^{\circ}$; wind NW $20 \mathrm{mph}$; 4 inches of snow. 10 species, 237 individuals. Sharp-tailed Grouse, 12; Ring-necked Pheasant, 6; Gray Partridge, 8; Great Horned Owl, 1; Snowy Owl, 1; Downy Woodpecker, 2; Black-billed Magpie, 18; Black-capped Chickadee, 6; House Sparrow, 8; Snow Bunting, 175.-W. W. Riome.

DESCHAMBAULT LAKE, Sask. Jan. 2,$1966 ; 3 \frac{1}{2}$ hours and 4 miles on foot about the settlement; temp. $-20^{\circ}$ to $-11^{\circ}$; wind $\mathrm{W}$ at $9 \mathrm{mph}$; clear. 6 species, 103 individuals. Black-billed Magpie, 2; Common Raven, 2; Blackcapped Chickadee, 1; Boreal Chickadee, 4; Common Redpoll, 90; Whitewinged Crossbill, 4. - Richard M. Sanderson.

DILKE, Sask. Jan. 1, 1966; 35 miles by car and $3 \frac{1}{2}$ miles on foot in 5 hours plus choring around farmyard; temp. $-10^{\circ}$ to $-15^{\circ}$; wind $\mathrm{NW} 5 \mathrm{mph} ; 12$ inches of snow. 8 species, 302 individuals. Gray Partridge, 32; Snowy Owl, 1; Horned Lark, 3; Black-billed Magpie, 15; House Sparrow, 40; Rusty Blackbird, 6; Common Redpoll, 18; Snow Bunting, 187. (Add: Sharptailed Grouse, Dec. 22 and 27; Rock Dove, Dec. 24.) - Boswell Belcher (compiler), 'Margaret Belcher, Mr. and Mrs. S. R. Belcher.

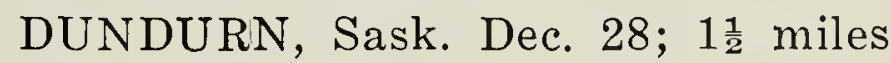
on foot in 1 hour in open country, bluffs and farmyards; temp. $-10^{\circ}$; wind ENE at $5 \mathrm{mph}$; overcast and snowing; 6 inches of snow on ground. 3 species, 58 individuals. Black-billed Magpie,3; Black-capped Chickadee, 1; House Sparrow, 54. (Add: Gray Partridge, 1, Dec. 30; Downy Woodpecker, 1, Jan. 1; Snow Bunting, 50, Dec. 30.) -Harry Harder.

ERINFERRY, Sask. Jan. 2, 1966; temp. $-20^{\circ}$; calm. 13 species, 70 individuals. Hairy Woodpecker, 2; Downy 
Woodpecker, 2; Gray Jay, 2; Blue Jay, 8; Black-billed Magpie, 2; Common Raven, 4; Black-capped Chickadee, 8; Boreal Chickadee, 2; Evening Grosbeak, 6; Pine Grosbeak, 4; Common Redpoll, 12; Red Crossbill, 6; Snow Bunting, 12.-Mrs. E. A. Dodd.

ETHELTON, Sask. Jan. 2, 1966; 20 miles by car and around yard; temp. $-20^{\circ}$; clear and calm. 9 species, 140 individuals. Snowy Owl, 1; Hairy Woodpecker, 1; Black-billed Magpie, 6; Common Raven, 2; Black-capped Chickadee, 3; House Sparrow, 37; Evening Grosbeak, 20; Common Redpoll, 5; Snow Bunting, 65. (Add: Gray Partridge, 12, Dec. 27; Saw-whet Owl, 1, Jan. 1.)-Genevieve Belliveau (compiler), Mr. C. S. Campbell, Mrs. G. Clarke.

FORT QU'APPELLE, Sask. Jan. 1, 1966 ; temp. $-15^{\circ}$; about 12 inches of snow. 9 species, 49 individuals. Sharptailed Grouse, 2; Great Horned Owl, 1; Hairy Woodpecker, 1; Black-billed Magpie, 1; Black-capped Chickadee, 12; Robin, 1; Bohemian Waxwing, 15; House Sparrow, 3; Pine Grosbeak, 12; Harris' Sparrow, 1. (Add: Gray Partridge, 15, Dec. 25 and 6, Jan. 2; Rock Dove, 16, Dec. 26 and 29; Downy Woodpecker, 1, Dec. 26 and 30; Blue Jay, 2, Dec. 22 and 1, Dec. 31, and Jan. 2; Northern Shrike, 1, Dec. 28; Common Redpoll, 200, Dec. 24; Slatecolored Junco, 1, Dec. 23 and 1, Dec. 29; Snow Bunting, 15, Dec. 25.)-Dr. and Mrs. G. D. Barnett, E. Manley Callin (compiler), Richard Carter, Mr. and Mrs. Errol Cochrane, Mr. and Mrs. Wm. Gray, Richard Nevard, Horace Reed, Mr. and Mrs. Jos. Rumancik.

HAWARDEN, Sask. Dec. 29; 20 miles by car and around farm yard; $7 \frac{1}{2}$ hours; temp. $-2^{\circ} ; 6$ inches of snow. 4 species, 510 individuals. Gray Partridge, 8; Horned Lark, 300; Blackbilled Magpie, 2; House Sparrow, 200. (Add: Snowy Owl, 1, Dec. 31; Bohemian Waxwing, 1, Jan. 1; Snow Bunting, 200, Dec. 31 and 1, Jan. 1.)Harold Kvinge.

INDIAN HEAD, Sask. Dec. 23; 16 miles by car and 7 miles on foot; 7 hours; temp. $5^{\circ}$; no wind; clear, except for fog in low areas; 10 inches of snow, 9 species, 156 individuals. Sharptailed Grouse, 2; Gray Partridge, 7; Rock Dove, 8; Hairy Woodpecker, 3; Horned Lark, 2; Black-billed Magpie, 6; Black-capped Chickadee, 3; House Sparrow, 120; Common Redpoll, 5. (Add: Snowy Owl, 1, Jan. 2; Snow Bunting, 57, Jan. 2.)-Lorne Scott.

KELVINGTON, Sask. Jan. 1, 1966; 1 hour about the town; temp. $-20^{\circ}$; clear. 5 species, 175 individuals. Blackbilled Magpie, 10; Common Raven, 1; Black-capped Chickadee, 3; House Sparrow, 86; Evening Grosbeak, 75. (Add: Hairy Woodpecker, 1, Dec. 24.) -Steve, Verna and Brian Waycheshen.

KINISTINO, Sask. Dec. 30; 36 miles; 2 hours on foot and $3 \frac{1}{2}$ hours by car; temp. $-15^{\circ}$ to $-10^{\circ}$; wind at $10 \mathrm{mph}$; snowing in afternoon,. 10 species, 582 individuals. Sharp-tailed Grouse, 7; Rock Dove, 7; Snowy Owl, 1; Boreal Owl, 1; Downy Woodpecker, 1; Blackbilled Magpie, 7; Common Raven, 9; Black-capped Chickadee, 2; House Sparrow, 27; Snow Bunting, 520.Peter Wojcichowsky.

McLEAN, Sask. Dec. 24; 5 species, 50 individuals. Hairy Woodpecker, 2; Downy Woodpecker, 2; Black-billed Magpie, 2; Black-capped Chickadee, 20; Pine Grosbeak, 24. (Add: Sharptailed Grouse, 6, Dec. 31; Gray Partridge, 6, Dec. 31).-Mrs. Harold Bray, Mrs. Hilda Newton.

MOOSE JAW, Sask. Dec. 27; 63 miles by car and 8 miles on foot; temp. $-19^{\circ}$ to $-15^{\circ}$; calm; 4 inches of snow. 10 species, 415 individuals. Ring-necked Pheasant, 30; Gray Partridge, 19; Rock Dove, 117; Great Horned Owl, 1; Black-billed Magpie, 25; Blackcapped Chickadee, 5; Bohemian Waxwing, 6; House Sparrow, 200; Pine Grosbeak, 8; Common Redpoll, 4. (Add: Downy Woodpecker, 2, Dec. 24; Starling, 2, Dec. 31; Slate-colored Junco, 6, Dec. 29.)-Mr. and Mrs. G. P. Brodie, John Ellis, Mrs. F. V. Humphreys, Miss Patricia Kern, Mr. and Mrs. Cy Knight, Michael Rhodes, Mrs. George Rhodes, Mrs. C. V. 
Stokins, Mrs. F. B. Taylor (compiler), Mr. and Mrs. Clarence West (Moose Jaw Natural History Society).

NIPAWIN, Sask. Dec. 27; $6 \frac{1}{4}$ hours; temp. $-25^{\circ}$; windy. 20 species, 430 individuals. Ruffed Grouse, 1; Sharptailed Grouse, 5; Gray Partridge, 8; Pileated Woodpecker, 1; Hairy Woodpecker, 1; Downy Woodpecker, 1; Gray Jay, 3; Blue Jay, 9; Black-billed Magpie, 8; Common Raven, 16; Blackcapped Chickadee, 5; Boreal Chickadee, 2; Red-breasted Nuthatch, 1; Bohemian Waxwing, 34; Northern Shrike, 1; House Sparrow, 250; Evening Grosbeak, 19; Pine Grosbeak, 23; Common Redpoll, 12; Snow Bunting, 30. (Add: Goshawk, 1, Dec. 30; Mourning Dove, 1, Dec. 15.)-Maurice G. Street.

PIAPOT, Sask. Jan. 7, 1966; about farmyard and 16 miles by car. 22 species, 3744 individuals. Goshawk, 2; Rough-legged Hawk, 1; Golden Eagle, 3; Bald Eagle, 1; Prairie Falcon, 1; Sharp-tailed Grouse, 17; Ring-necked Pheasant, 2; Gray Partridge, 14; Great Horned Owl, 3; Hairy Woodpecker, 1 ; Downy Woodpecker, 2 ; Horned Lark, 70; Black-billed Magpie, 18; Black-capped Chickadee, 14; Robin, 1; Bohemian Waxwing, 27; Northern Shrike, 2; House Sparrow, 94; Rusty Blackbird, 1; Pine Grosbeak, 70; Common Redpoll, 400; Snow Bunting, 3000. -Steve A. Mann (compiler), Peter Swain.

REGINA, Sask. Dec. 27; 231 miles in 21 hours by car and $18 \frac{1}{2}$ party miles in 17 hours on foot; temp. $-25^{\circ}$ to $-15^{\circ}$; wind W $8 \mathrm{mph}$ to $\mathrm{NE} 3 \mathrm{mph} ; 6$ inches of snow; clear except mist over Waterfowl Park. 31 species, 3538 individuals. Mute Swan, 4; Whistling Swan, 4; Canada Goose, 200; Mallard, 412 ; Lesser Scaup, 4; Common Goldeneye, 1; Sharp-tailed Grouse, 5; Ring-necked Pheasant, 1; Gray Partridge, 126; American Coot, 3; Rock Dove, 40; Great Horned Owl, 6; Snowy Owl, 5; Hairy Woodpecker, 1; Downy Woodpecker, 3; Black-billed Magpie, 41; Black-capped Chickadee, 17; Boreal Chickadee, 3; Brown Creeper, 1; Robin, 8; Bohemian Wax- wing, 31; Cedar Waxwing, 1; Northern Shrike, 1; Starling, 25; House Sparrow, 1797; Rusty Blackbird, 1; Pine Grosbeak, 92; Common Redpoll, 285; Slate-colored Junco, 1; Whitethroated Sparrow, 1; Snow Bunting, 418. (Add: Canvasback, 1, Dec. 21; Ruddy Duck, 6, Dec. 21; Pigeon Hawk, 1, Dec. 31; Red-breasted Nuthatch, 2, Dec. 28; Evening Grosbeak, 1, Jan. 1; Purple Finch, 1, Dec. 28.)-Mr. and Mrs. George Allen, Mrs. J. B. Bailey, Margaret Belcher, D. G. Bobbitt, Frank Brazier, E. H. Cooke, Mrs. Elizabeth Cruickshank, Elmer Fox, Jean Freeman, Doug Gilroy, James Jowsey, Harriet Jowsey, Elizabeth Lane, Frances Lane, Mr. and Mrs. Frank Lane, Dr. and Mrs. George Ledingham, Helen Morrison, Sally Moss, Robert Nero, Maureen Rever, Richard Sanderson, Mr. and Mrs. Hugh Smith (compilers) (Regina Natural History Society).

SASKATOON, Sask. Dec. 27; 17 party miles on foot in $14 \frac{1}{2}$ hours and 214 by car in 26 hours; temp. $-31^{\circ}$ to $-22^{\circ}$; wind SW $10 \mathrm{mph}$; clear; 3 inches of snow. 25 species, 4395 individuals. Mallard, 15; Pigeon Hawk, 1; Ruffed Grouse, 1; Sharp-tailed Grouse, 53; Ring-necked Pheasant, 5; Gray Partridge, 141; Rock Dove, 315; Great Horned Owl, 1; Hairy Woodpecker, 4; Downy Woodpecker, 2; Horned Lark, 3; Blue Jay, 6; Black-billed Magpie, 186; Black-capped Chickadee, 25; Redbreasted Nuthatch, 1 ; Bohemian Waxwing, 381; Cedar Waxwing, 6; Northern Shrike, 2; Starling, 60; House Sparrow, 1836; Evening Grosbeak, 11; Pine Grosbeak, 204; Hoary Redpoll, 35; Common Redpoll, 794; Snow Bunting, 307.-John Black, W. J. Brucks, John Crabb, Don Dabbs, Bob Folker, Jonathan Gerrard, Dr. and Mrs. J. B. Gollop, Colin Gregory, Mr. and Mrs. Vic Harper, Jim Hogg, Dr. and Mrs. Stuart Houston, Stan Houston, Dr. Toshio Ishikawa, Mr. and Mrs. Murray Martin, John and Stanley Shadick, Dale Shockey, Jim Slimmon, Al Smith, Jim Wedgwood (Saskatoon Natural History Society).

SHAUNAVON, Sask. Dec. 28; 85 
miles by car in $5 \frac{1}{2}$ hours; temp. $-15^{\circ}$; calm; overcast; 2 inches of snow. 13 species, 384 individuals. Rough-legged Hawk, 2; Golden Eagle, 2; Ringnecked Pheasant, 2; Gray Partridge, 13; Rock Dove, 1; Long-eared Owl, 1; Short-eared Owl, 1; Horned Lark, 277; Black-billed Magpie, 18; House Sparrow, 1; Rusty Blackbird, 11; Common Redpoll, 2; Snow Bunting, 53.-J. David Chandler.

SPIRIT LAKE, Sask. Dec. 21; 5 hours and 4 miles on foot, as well as farmyards and 2 feeding stations; temp. $30^{\circ}$; wind NW $15 \mathrm{mph}$; cloudy in a.m., clear in p.m.; 6 inches of snow in woods. 10 species, 182 individuals. Ruffed Grouse, 7; Hairy Woodpecker, 3; Downy Woodpecker, 5; Black-billed Magpie, 3; Black-capped Chickadee, 30 ; Bohemian Waxwing, 30; House Sparrow, 25; Evening Grosbeak, 12; Pine Grosbeak, 7; Snow Bunting, 60. (Add: Sharp-tailed Grouse, 4, Dec. 22; Rock Dove, 6, Dec. 24; Common Redpoll, 5, Dec. 31.)-Wm. Anaka, Joyce Gunn.

WOLSELEY, Sask. Dec. 31; 20 miles on country roads; temp. $0^{\circ} ; 16$ inches of snow. 7 species, 117 individuals. Gray Partridge, 9; Snowy Owl, 1; Black-billed Magpie, 3; Black-capped Chickadee, 1; Northern Shrike, 1; House Sparrow, 100; Pine Grosbeak, 2. (Add: Ruffed Grouse, 2, Dec. 23; Horned Lark, 2, Jan. 1; Snow Bunting, 1, Jan. 1).-Don Hayward.

WOODROW, Sask. Dec. 22; 41 miles by truck around the lake bottom on west side of the Wood River; temp. $30^{\circ}$; calm; 1 inch of snow. 12 species, 191 individuals. Rough-legged Hawk, 1; Golden Eagle, 1; Ring-necked Pheasant, 5; Gray Partridge, 21; Rock Dove, 6; Great Horned Owl, 1; Snowy Owl, 2; Short-eared Owl, 1; Horned Lark, 30; Black-billed Magpie, 3; House Sparrow, 40; Snow Bunting, 80. -C. H. Shulver.

YELLOW CREEK, Sask. Dec. 31; 4 miles on foot in 3 hours; temp. $-8^{\circ}$; cloudy; 24 inches of snow. 4 species, 41 individuals. Ruffed Grouse, 3; Downy Woodpecker, 1; Bohemian Waxwing, 35; Common Redpoll, 2.
(Add: Evening Grosbeak, 10, Jan. 1; Pine Grosbeak, 15, Jan. 1.)-Derwent Mazur.

YELLOW CREEK, Sask. Jan. 2, 1966; $4 \frac{1}{2}$ miles on foot in 3 hours through farmyards, fields and light brush; temp. $-10^{\circ}$ to $-5^{\circ}$; wind light, becoming gusty; overcast with light snow; 13 inches of snow. 6 species, 203 individuals. Hairy Woodpecker, 2; Downy Woodpecker, 1; Black-capped Chickadee, 3; House Sparrow, 68; Pine Grosbeak, 36; Snow Bunting, 93. (Add: Great Horned Owl, 1, Dec. 21 and 23; Black-billed Magpie, 1, Dec. 31; Northern Shrike, 1, Jan. 1; Common Redpoll, 50, Dec. 31.)-Bohdan Pylypec.

WETASKIWIN, Alta. Dec. 25; 14 miles by car; temp. $-15^{\circ}$; wind $\mathrm{NW}$ $20 \mathrm{mph}$; clear with drifting snow; 6 inches of snow. 4 species, 152 individuals. Black-billed Magpie, 1; Blackcapped Chickadee, 6; House Sparrow, 20; Snow Bunting, 125. (Add: Roughlegged Hawk, 1, Dec. 21; Great Horned Owl, 1, Jan. 2; Hairy Woodpecker, 1, Dec. 21; Downy Woodpecker, 1, Dec. 26; Blue Jay, 1, Dec. 26; Northern Shrike, 1, Dec. 30; Pine Grosbeak, 1, Dec. 26.)—Fred H. Pegg.

CAMBRIDGE BAY, N.W.T. Dec. 25; 14 miles in 1 hour by car and 4 miles in $3 \frac{1}{2}$ hours on foot; temp. $-40^{\circ}$. to $-36^{\circ}$; wind WNW $16 \mathrm{mph}$; no sun; 6 inches of snow. 1 species, 5 individuals. Willow Ptarmigan, 5. - Ramon C. Burron (compiler), Rowan Raphael.

\section{COOPERATIVE SPRING MIGRATION STUDY}

Records are again requested for the continent-wide survey being made under the auspices of the U.S. Fish and Wildlife Service. As soon as it is available, we shall have a list of species for which reports are wanted; please write for the list and then submit your records by June 15, 1966, to

Mrs. MARY HOUSTON

863 University Drive, Saskatoon, Sask. 